\title{
Zehn-Punkte-Plan zur Vermeidung behandlungsassoziierter Infektionen und Antibiotika-Resistenzen
}

\author{
Winfried V. Kern
}

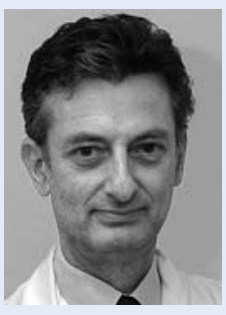

Winfried V. Kern
„D ie Bundesregierung hat in den vergangenen Jahren sowohl die bestehenden Gesetze und Instrumente ausgebaut, als auch neue Maßnahmen und Angebote entwickelt, um die Entstehung von behandlungsassoziierten Infektionen und Antibiotika-Resistenzen zu verringern, doch sind die bisherigen Erfolge noch nicht zufriedenstellend“ [1]. Gerade noch rechtzeitig zum G7-Gipfel (nota bene: eigentlich ja ein „Weltwirtschaftsgipfel“) wurden der Zehn-Punkte-Plan [1] und das Dokument zu DART II (DART 2020) [2] fertig gestellt.

Besonders viele Reaktionen gab es eigentlich nicht. Mehr oder weniger wurden in den Meldungen und Kommentaren die zehn Punkte abgeschrieben, manchmal versehen mit knappen Zusatzkommentaren. Sehr „investigativ“ und etwas dümmlich: „Recherchen von ZEIT ONLINE, DIE ZEIT und CORRECT!V hatten erst kürzlich ergeben, dass allein in Deutschland jedes Jahr wohl Tausende mehr Menschen an den Folgen solcher Keiminfektionen sterben als offiziell bekannt“ [3]. Nicht besonders originell: „ein Problem: anders als zum Beispiel in den Niederlanden gibt es hierzulande kein routinemäßiges Screening“ " 4 ]- warum auch ? „Gröhe will nun die Experten des Robert-Koch-Instituts (RKI) stärker in die Kontrolle der Kliniken einbeziehen“ [5] - na dann, wir freuen uns schon darauf - statistisch ist das großzügig gerechnet ein halber, vielleicht auch mal ein ganzer Arbeitstag pro Jahr pro Klinik. O lala: „nach wie vor problematisch ist allerdings die Verfügbarkeit von Hygienepersonal auf dem Arbeitsmarkt“ erklärte die Deutsche Krankenhausgesellschaft sehr kritisch [6]. Hier sei es „wichtig, die Instrumente des Personalförderprogramms nachzujustieren, insbesondere dieses Programm zu verlängern und darauf hinzuwirken, dass die Krankenkassen den Krankenhäusern die Bewilligung von Fördermitteln weniger restriktiv gewähren“ [6].

Wie geht es nun eigentlich weiter? Der G7-Gipfel ist ja vorbei. Grexit ist wichtiger. Und es gibt ja auch noch MERS und schon wieder ein paar Ebola-Fälle. Was sagen die Fachgesellschaften? Was sagen wir dazu?
Was ist Ihr persönlicher Zehn-Punkte-Plan? Unter den Fachgesellschaften ist es die DGI, die dazu direkt Stellung genommen hat in einer Pressemitteilung [7], und es war die DGKH, die einen eigenen kommentierten Neun-Punkte-Plan veröffentlicht hat (allerdings bereits vor dem Gröhe-Papier) [8], kein öffentlicher Kommentar der DGHM oder einer anderen relevanten Fachgesellschaft im Bereich der Infektionsmedizin. In den beiden Stellungnahmepapieren der DGI und DGKH geht es um Investitionen - in Pflegepersonal, Arztstellen, Lehrstühle, Fort- und Weiterbildung und Krankenhausbau. Also Geld. Gut so! Also war es doch richtig, beim und zum Wirtschaftsgipfel diese Diskussion anzustacheln.

Man merkt es eben nun allenthalben und überall. Ohne Moos nichts los. Dabei hätte das alles so schön funktionieren können. Mit dem so genannten Hygieneförderprogramm ( $\$ 4$ Abs. 11 Krankenhausentgeltgesetz) sollten über 300 Millionen $€$ für solche Investitionen in Personal und Fort- und Weiterbildung verfügbar gemacht werden (!!!) - verbunden mit der Hoffnung auf eine Verstetigung nach Auslaufen des Programmes. Die Finanzmittel dafür sollten vom Krankenhaus mit den Krankenkassen vor Ort jährlich als prozentualer Aufschlag auf den Gesamtbetrag der Erlöse ausgezahlt werden - nach Ausweisung dieser Finanzmittel und „Rechnungstellung“ durch das Krankenhaus. Fragen Sie mal, was daraus geworden ist! > 300 Millionen $€$ ! Quasi nichts ist daraus geworden! Wie in Griechenland! Wo ist das Geld nur? Von überall hört man, die Verhandlungen mit den Kassen seien schwierig und in der Regel erfolglos - keine Millionen, kein Euro, kein Cent. Und nächstes Jahr läuft das Programm eigentlich aus. Schon wieder wie Griechenland! Ein nächstes Programm muss her? Aber man kann die „alten“ Millionen ja vielleicht irgendwo wieder finden und vielleicht sogar mit Guthabenzinsen wieder einsetzen?

Wir von der DGI haben ausgerechnet, dass man in drei Jahren 300 Infektiologen weiterbilden könnte, die mit ein paar Extramonaten auch noch Kurse und Praktikum für Krankenhaushygiene ableisten könnten - wirklich! 
Das ginge! Es würde 40 bis 50 Millionen $€$ kosten stimmt, das ist ja viel weniger als die $>300$ verschwundenen Millionen! Fast unglaublich. Es ginge schnell! Und es könnte ganz unkompliziert ablaufen! Die Krankenhäuser müssten sich direkt dafür bewerben können und würden dann Kollegen dafür abstellen und abordnen und das Geld als Personalersatz in dieser Zeit verwenden. Die dann an ihre Entsendeklinik zurück kehrenden geschulten (Infektiologie incl. Antibiotic Stewardship [ABS] plus Krankenhaushygiene) Kolleginnen und Kollegen würden zugleich einen Qualitätspunkt darstellen. Es dürften natürlich nur kleinere und mittlere Kliniken sich dafür bewerben, die bisher keinerlei qualifizierte Kräfte vor Ort beschäftigt haben und seit Längerem sehnsüchtig auf einen ABS-Platz warten und ungeduldig gerne einen Hygieniker vor Ort hätten lieber, als eine externe Hygieneberatung kaufen zu müssen.

Diese zusätzliche „Workforce“ wäre prima. Wir haben nämlich tatsächlich sehr wenig infektionsmedizinische Experten in den $~ 1800$ Akutkliniken in Deutschland: 300 Mikrobiologen, <100 Hygieniker, 300 Infektiologen, vielleicht 100 - 200 ärztliche ABS-Experten (die nicht zugleich Mikrobiologe, Hygieniker oder Infektiologe sind) und vielleicht 50-100 Apotheker mit ABSExperten-Zertifikat.

Vielleicht sollten wir auch abstimmen. Wie die Griechen. Auch, wenn gar kein konkretes Angebot der Verhandlungspartner auf dem Tisch liegt. Wir stimmen einfach über unseren eigenen Vorschlag ab. Vielleicht auch - wie die Griechen - unter Hinweis auf unsere Würde, die wir alles immer noch besser machen sollen und die Millionen Investitionen aber irgendwie nicht an der richtigen Stelle ankommen wollen. Unser Slogan: „Grexit (Glykopeptid-resistente Enterokokken raus!) et al. - wir machen es besser für weniger“ oder „Aus Zehn mach’ Eins, aber richtig!“

\section{Quellen}

1 https://www.bundesgesundheitsministerium.de/ministerium/meldungen/2015/10-punkte-plan-zu-antibiotika-resistenzen.html

2 http://www.bmg.bund.de/fileadmin/dateien/Publikationen/ Ministerium/Broschueren/BMG_DART_2020_Bericht_dt.pdf

3 http://www.zeit.de/wissen/gesundheit/2015-03/mrsa-multiresistente-erreger-hermann-groehe-krankenhaeuser

4 http://www.spiegel.de/gesundheit/diagnose/mrsa-und-cozehn-punkte-plan-gegen-krankenhauskeime-a-1025148.html

5 http://www.sueddeutsche.de/gesundheit/zehn-punkte-planvon-gesundheitsminister-groehe-kampf-den-killer-keimen1.2404562

6 http://www.aerzteblatt.de/nachrichten/62254/Groehe-legt10-Punkte-Plan-zur-Bekaempfung-resistenter-Keime-vor? $s=$ Zehn-Punkte-Plan

7 http://www.dgi-net.de/files/Presse/Pressemeldung_10-Punkte_01-04-2015.pdf

8 http://www.krankenhaushygiene.de/pdfdata/hm/ 2015_HM_01-02_Aktuelle_Forderungen_der_DGKH.pdf

9 http://www.bundesaerztekammer.de/downloads/Stat14AbbTab2.pdf

10 http://dipbt.bundestag.de/dip21/btd/18/036/1803600.pdf 